\title{
Hyperbaric oxygen therapy effects on pulmonary functions: a prospective cohort study
}

\author{
Amir Hadanny ${ }^{1,2,3^{*}}$ (D), Tal Zubari ${ }^{4}$, Liat Tamir-Adler ${ }^{4}$, Yair Bechor ${ }^{1}$, Gregory Fishlev ${ }^{1}$, Erez Lang ${ }^{1}$, Nir Polak? \\ Jacob Bergan ${ }^{4}$, Mony Friedman ${ }^{4}$ and Shai Efrati ${ }^{1,2,5,6}$
}

\begin{abstract}
Background: Oxygen toxicity is one potential side effect of hyperbaric oxygen therapy (HBOT). Previous small studies showed mild reductions in pulmonary functions reflecting reductions in small airway conductance after repetitive hyperbaric oxygen sessions. However, there are no updated data with well performed pulmonary tests that address the pulmonary effect of the currently used HBOT protocols.

The aim of this study was to evaluate the effect of HBOT on pulmonary functions of patients receiving the currently used HBOT protocol.
\end{abstract}

Methods: Prospective analysis included patients, 18 years or older, scheduled for 60 daily HBOT sessions between 2016 and 2018. Each session was 90 min of 100\% oxygen at 2 ATA with 5 min air breaks every 20 min, 5 days per week. Pulmonary functions, measured at baseline and after HBOT, included forced vital capacity (FVC), forced expiratory volume in $1 \mathrm{sec}$ (FEV1) and peak expiratory flow rate (PEF).

Results: The mean age was $60.36 \pm 15.43$ and 62.5\% (55/88) were males. Most of the patients (83/88, 94.3\%) did not have any pulmonary disease prior to inclusion and $30.7 \%$ (27/88) had a history of smoking.

Compared to baseline values, at the completion of 60 HBOT sessions, there were no significant changes in FEV1 (0.163), FEV1/FVC ratio (0.953) and FEF25-75\% (0.423). There was a statistically significant increase though not clinically relevant increase in FVC $(0.1 \pm 0.38 \mathrm{I})$ and PEF $(0.5 \pm 1.4 \mathrm{I})$ with a 0.014 and 0.001 respectively.

Conclusion: Regarding pulmonary functions, repeated hyperbaric oxygen exposure based on the currently used HBOT protocol is safe. Surprisingly, there was a modest non clinically significant though statistically significant improvement in PEF and FVC in the current cohort of patients who were without chronic lung diseases.

Trial registration: Clinicaltrials.gov, trial ID: NCT03754985, (Nov 2018) Retrospectively registered.

Keywords: Pulmonary function, Oxygen toxicity, Hyperbaric oxygen, HBOT, PFT

\section{Take home message}

- Repeated hyperbaric oxygen sessions of 90 min at 2 ATA, with $5 \mathrm{~min}$ air breaks every $20 \mathrm{~min}$, are safe and have no negative impact on pulmonary functions.

\footnotetext{
* Correspondence: Amir.had@gmail.com

${ }^{1}$ The Sagol Center for Hyperbaric Medicine and Research, Shamir Medical Center, Zerifin, Israel

${ }^{2}$ Sackler School of Medicine, Tel-Aviv University, Tel-Aviv, Israel

Full list of author information is available at the end of the article
}

- The currently used HBOT protocol induces a modest improvement in PEF and FVC in patients without chronic lung diseases.

\section{Background}

Oxygen is a vital but potent biochemical and as such, it can be toxic depending on dose and exposure duration [1]. Although oxygen-induced damage has been reported in most tissues, the lungs are mostly affected since the oxygen partial pressure $\left(\mathrm{PO}_{2}\right)$ is the highest in the alveoli. Pulmonary oxygen toxicity manifests as two overlapping phases as seen in pathology. The acute exudative

(c) The Author(s). 2019 Open Access This article is distributed under the terms of the Creative Commons Attribution 4.0 International License (http://creativecommons.org/licenses/by/4.0/), which permits unrestricted use, distribution, and 
phase includes interstitial and alveolar edema, hemorrhage, inflammation and fibrinous exudate along with injury to the endothelial cells and type I alveolar cells. The subacute proliferative phase is defined by interstitial fibrosis, fibroblastic proliferation, and hyperplasia of type II alveolar cells [2]. The first sign of pulmonary toxicity is tracheobronchial irritation, which is clinically expressed as substernal or pleuritic pain [1] followed by a decrease in pulmonary function in both the acute and subacute proliferative phase [3].

Clarke et al.'s novel studies show that the incidence of pulmonary oxygen toxicity increases both with the inspiratory partial pressure and the time of exposure in a continuous single exposure $[4,5]$. In normal humans, toxicity can be expected after about $10 \mathrm{~h}$ of $100 \%$ oxygen at 1ATA, after $8-14 \mathrm{~h}$ at 1.5 ATA and $3-6 \mathrm{~h}$ at 2 ATA of continuous exposure with symptoms subsiding after 4 hours [6].

Hyperbaric oxygen therapy (HBOT), which utilizes both high pressure and high concentrations of oxygen, in multiple daily sessions per patient, has the potential to induce pulmonary oxygen toxicity. Two previous studies evaluated the effects of repetitive HBOT on pulmonary function. Pott et al. [7] studied 14 patients who underwent 30 daily sessions of $90 \mathrm{~min}$ exposure to 2.4 ATA pure oxygen in a monoplace chamber. Patients were exposed to hyperoxia without any air breaks during the sessions. There were no significant changes in forced vital capacity (FVC) or diffusing capacity. Furthermore, most of the patients in this study were heavy smokers with impaired diffusing capacity at baseline. Thorsen et al. [8] included 20 patients who underwent 21 repetitive daily HBOT sessions. The protocol included $90 \mathrm{~min}$ of 2.4 ATA $100 \%$ oxygen in 3 cycles of $30 \mathrm{~min}$ each, separated by two 5 min breaks where patients breathed air in-between ("air breaks"). There were no significant changes in FVC, forced expiratory flow at $25 \%$ of pulmonary volume (FEF25\%) or peak expiratory flow rate (PEF). However, there were significant reductions in forced expiratory volume in 1 second (FEV1). No significant change was noticed in diffusing capacity.

In recent years, HBOT is used for a growing number of patients for new indications, using protocols that are based on lower oxygen pressure (2 ATA or less), but for a prolonged period with more daily sessions (40-60 sessions). The new emerging indications are mostly neurological, including idiopathic sudden sensorineural hearing loss [9], post-stroke and post-traumatic brain injury [10-12], post-radiation injury, as well as chronic pain such as fibromyalgia syndrome [13]. In these conditions, the protocols are based on 40-60 HBOT daily sessions. The safety of these longer protocols regarding pulmonary oxygen toxicity hasn't been well evaluated yet.
The aim of the current study was to assess pulmonary oxygen toxicity as measured by pulmonary functions associated with 60 daily sessions of HBOT.

\section{Methods}

The study was performed as a prospective cohort study conducted at the Sagol Center for Hyperbaric Medicine and Research at Assaf Harofeh Medical Center between February 2016 and June 2018. The protocol was approved by our institution's institutional review board (IRB) (0024-16-ASF). All participants signed written informed consent prior to their inclusion.

\section{Participants}

The study included participants 18 years or older, scheduled for $60 \mathrm{HBOT}$ sessions for any indication. Active smokers were excluded but patients who quit smoking more than 6 months prior to inclusion were allowed in the study.

Exclusion criteria included active smoking, severe known pulmonary disease, chest pathology incompatible with HBOT, inner ear disease, claustrophobia, other neurological conditions, pregnancy, previous HBOT within 6 months prior to inclusion and the inability to sign informed consent. Patients who did not complete 60 sessions due to non-pulmonary reasons were excluded as well.

Data collected from the patient's medical files included age, gender, chronic medical conditions, medications, previous smoking and indication for HBOT therapy.

\section{Protocol}

After signing an informed consent form, the participants underwent a pulmonary function baseline evaluation. Participants were treated in a multiplace chamber (HAUXLife-Support $\mathrm{GmbH}$ ) for 60 daily sessions, 5 days a week. Each session consisted of 90 min of exposure to 100\% oxygen at 2 ATA with 5 min air breaks every $20 \mathrm{~min}$. Participants repeated their pulmonary function evaluation after the last HBOT session. Measurements were taken in the morning before entering the hyperbaric chamber of the first and last session (22-23 $\mathrm{h}$ post the previous session).

\section{Pulmonary function}

Measurements of pulmonary functions were performed using the MiniSpir testing apparatus (MIR- Medical International Research, USA). The equipment was calibrated using a 3-1 syringe before performing measurements according to the manufacturer's instructions. Measurements were performed by a trained technician. The forced expiratory maneuvers were performed as recommended by the guidelines [14].

The forced vital capacity (FVC), forced expiratory volume in $1 \mathrm{sec}$ (FEV1) and peak expiratory flow rate (PEF) 
were taken as the highest readings obtained from at least three satisfactory forced expiratory maneuvers. Mean forced mid-expiratory flow rate (FEF25-75\%) and forced expiratory flow rates at 25, 50 and $75 \%$ of FVC expired (FEF25\%, FEF50\% and FEF75\%) were taken as the best values from flow-volume loops not differing by $>5 \%$ from the highest FVC. Pulmonary function was analyzed using WinSpiroPRO with Knudson reference values [15].

\section{Sample size}

The expected mean changes in FVC in repeated spirometry of adults are $72 \pm 76 \mathrm{ml}$ [16]. Using alpha of 0.05 , the mean change in FVC in the current study with sample size of 88 patients gives a power of $93.3 \%$.

\section{Statistical analysis}

Continuous data were expressed as means \pm standard deviations. The normal distribution for all variables was tested using the Kolmogorov-Smirnov test. Dependent $\mathrm{t}$ tests were performed to compare changes within groups. Possible covariates (age, gender, biometric data, chronic medical conditions, medications) were analyzed using a general linear model. Categorical data were expressed in numbers and percentages and compared by chi-square tests. Univariate analysis was performed using ChiSquare/Fisher's exact test (where appropriate) or to identify significant variables $(P<0.05)$. The alpha level was set to 0.05 . Data were statistically analyzed using SPSS software (version 22.0).

\section{Results}

Between February 2016 and June 2018, 105 patients signed informed consents and performed baseline evaluations. Thirteen patients did not complete 60 HBOT sessions and were excluded from anaylsis. Three patients in whom one of the tests was performed without pre-test system calibration and one patient who did not complete the post HBOT evaluation were also excluded from final analysis. Accordingly, 88 patients were included in the final analysis (Fig. 1).

The mean age was $60.36 \pm 15.43$ and $62.5 \%(55 / 88)$ were males. Most of the patients $(83 / 88,94.3 \%)$ did not have any pulmonary disease prior to inclusion and $30.7 \%$ $(27 / 88)$ had a history of smoking. Most patients were treated for neurological indications $(71.6 \%, 63 / 88)$. There were significant differences in biometric measurements, chronic medical conditions, prescribed drugs and baseline pulmonary functions. See Table 1 for basic characteristics.

At baseline, FVC was $3.55 \pm 0.971$ or $96.15 \pm 19.33 \%$ of the predicted values and FEV1 was $2.83 \pm 0.731$ or $95.54 \pm 20.36 \%$. The FEV1/FVC ratio was $80.10 \pm 9.64$ or $101.56 \pm 13.56$ of the predicted values (Table 2). After HBOT, there were no significant changes in FEV1, the FEV1/FVC ratio and FEF25-75 (Table 2, $p>0.05$ ). There was a small statistically significant increase of $0.04 \pm$ $0.28 \mathrm{l}$ in $\mathrm{FVC}$, and $0.48 \pm 1.39 \mathrm{l} / \mathrm{min}$ in PEF $(p<0.05)$ (Table 2).

Age, gender, weight, height, BMI, chronic medical conditions, medications and HBOT indication were not associated with post HBOT FVC, FEV1, FEV1/FVC, FEF25-75 and PEF $(p>0.05)$. Pre HBOT-measurements were the only significant predictor for post HBOT values.

Clinically, none of the patients complained of any cough, irritation, dyspnea or chest pain during and post HBOT.

\section{Discussion}

In this currently largest prospective cohort study of 88 participants, repeated exposures of 60 daily sessions in 2

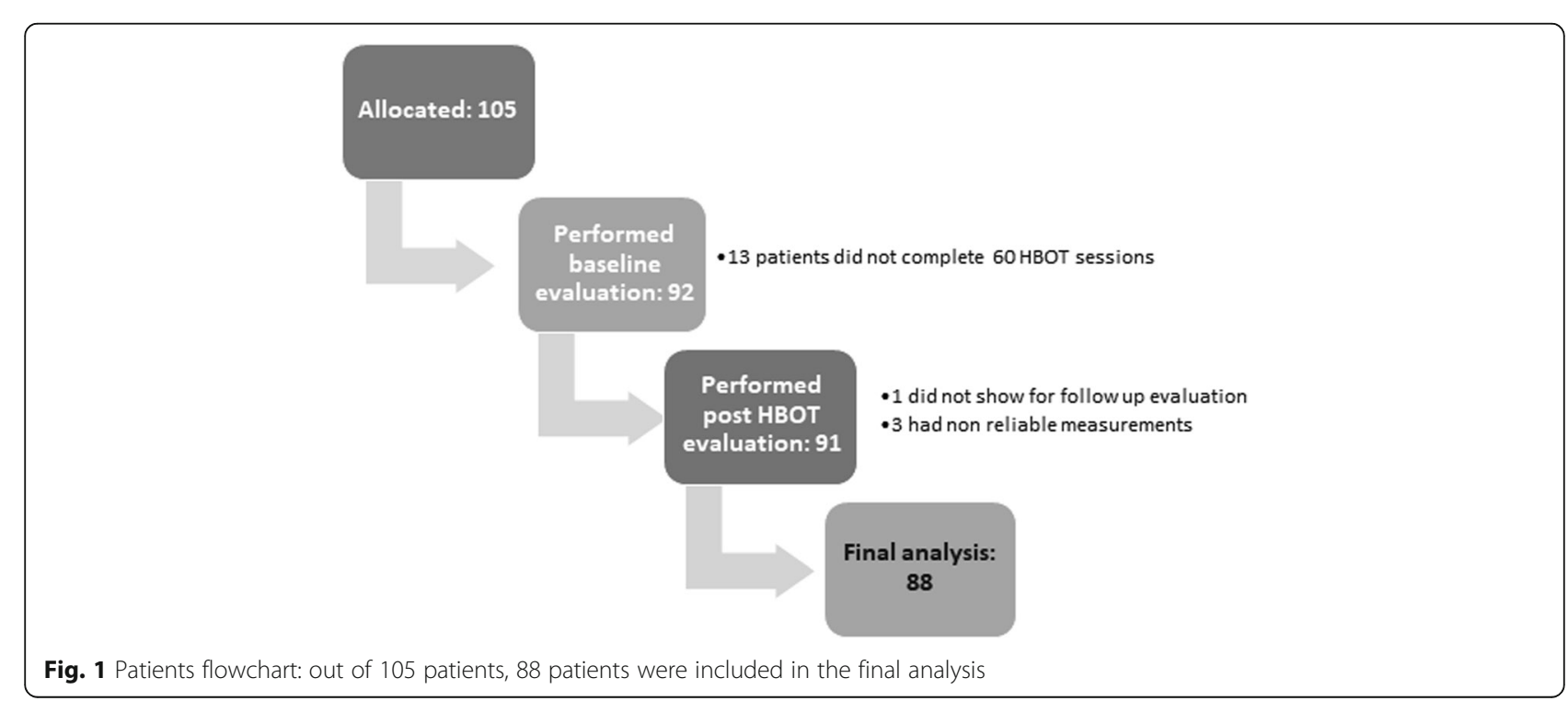


Table 1 Patients baseline characteristics

\begin{tabular}{|c|c|c|c|c|}
\hline & Total & Males & Females & Significance \\
\hline $\mathrm{N}$ & $88(100 \%)$ & $55(62.5 \%)$ & $33(37.5 \%)$ & \\
\hline Age (years) & $60.36 \pm 15.43$ & \pm 11.2665 .13 & $52.41 \pm 18.15$ & 0.0001 \\
\hline Height (cms) & $170.72 \pm 9.72$ & $175.63 \pm 6.54$ & $162.54 \pm 8.62$ & $<0.0001$ \\
\hline Weight (kgs) & $75.20 \pm 14.69$ & $81.94 \pm 13.16$ & $63.96 \pm 9.29$ & $<0.0001$ \\
\hline BMI & $25.67 \pm 3.79$ & $26.54 \pm 3.85$ & $24.25 \pm 3.28$ & 0.005 \\
\hline \multicolumn{5}{|l|}{ Chronic medical conditions } \\
\hline Diabetes mellitus & $17(19.3 \%)$ & $15(27.3 \%)$ & $2(6.1 \%)$ & 0.015 \\
\hline Hypertension & $26(29.5 \%)$ & $22(40 \%)$ & $4(12.1 \%)$ & 0.006 \\
\hline Hypercholesterolemia & $26(29.5 \%)$ & $21(38.2 \%)$ & $5(15.2 \%)$ & 0.022 \\
\hline Ischemic heart disease & $13(14.8 \%)$ & $13(23.6 \%)$ & 0 & 0.001 \\
\hline Pulmonary disease & $5(5.7 \%)$ & $5(9.1 \%)$ & 0 & 0.15 \\
\hline History of smoking & $27(30.7 \%)$ & $21(38.2 \%)$ & $6(18.8 \%)$ & 0.059 \\
\hline Pack years (in smokers) & $18.33 \pm 13.95$ & $19.31 \pm 15.15$ & $14.91 \pm 8.72$ & 0.51 \\
\hline Indication & & & & 0.707 \\
\hline Neurological & (65.9\%) 58 & $36(65.4 \%)$ & $22(66.6 \%)$ & \\
\hline Wounds/Radiation & $6(8.4 \%)$ & $3(5.5 \%)$ & $3(9.1 \%)$ & \\
\hline Other & $24(27.3 \%)$ & $16(29.0 \%)$ & $8(24.2 \%)$ & \\
\hline \multicolumn{5}{|l|}{ Medications } \\
\hline Anti-aggregation & 27 (30.7\%) & $21(38.2 \%)$ & $6(18.2 \%)$ & 0.049 \\
\hline ACE-Inhibitors & $17(19.3 \%)$ & $15(27.3 \%)$ & $2(6.1 \%)$ & 0.023 \\
\hline Statins & $34(38.6 \%)$ & $27(49.1 \%)$ & $7(21.2 \%)$ & 0.009 \\
\hline Proton pump inhibitors & $12(13.6 \%)$ & $9(16.4 \%)$ & $3(9.1 \%)$ & 0.336 \\
\hline \multicolumn{5}{|l|}{ Baseline Pulmonary functions } \\
\hline FEV1 & $2.83 \pm 0.73$ & $2.97 \pm 0.77$ & $2.60 \pm 0.61$ & 0.022 \\
\hline FVC & $3.55 \pm 0.97$ & $3.79 \pm 0.96$ & $3.15 \pm 0.87$ & 0.002 \\
\hline FEV1/FVC & $80.10 \pm 9.64$ & $78.44 \pm 10.1$ & $82.87 \pm 8.26$ & 0.036 \\
\hline PEF & $5.74 \pm 1.88$ & $6.87 \pm 2.0$ & $5.11 \pm 1.57$ & $<0.001$ \\
\hline
\end{tabular}

everything under 0.05 are in bold

Table 2 Pulmonary function pre and post HBOT

\begin{tabular}{lllll}
\hline & Baseline & Post HBOT & Mean Change & Significance \\
\hline FEV1 (I) & $2.83 \pm 0.73$ & $2.88 \pm 0.75$ & $0.04 \pm 0.28$ & 0.163 \\
Predicted (\%) & $95.54 \pm 20.36$ & $97.28 \pm 20.17$ & $1.74 \pm 9.90$ & 0.102 \\
FVC (I) & $3.55 \pm 0.97$ & $3.65 \pm 1.04$ & $0.1 \pm 0.38$ & $\mathbf{0 . 0 1 4}$ \\
Predicted (\%) & $96.15 \pm 19.33$ & $98.55 \pm 20.17$ & $2.40 \pm 9.95$ & $\mathbf{0 . 0 2 6}$ \\
FEV1/FVC & $80.10 \pm 9.64$ & $79.51 \pm 9.23$ & $(-0.60) \pm 7.21$ & 0.435 \\
Predicted (\%) & $101.56 \pm$ & $100.97 \pm 13.4$ & $(-0.59) \pm 9.01$ & 0.536 \\
& 13.56 & & & \\
FEF2575 (I) & $2.94 \pm 1.02$ & $2.89 \pm 1.00$ & $(-0.04 \pm 0.57)$ & 0.423 \\
Predicted (\%) & $93.86 \pm 31.10$ & $92.70 \pm 33.37$ & $(-1.15 \pm 20.37)$ & 0.597 \\
PEF (I/min) & $5.74 \pm 1.88$ & $6.23 \pm 2.03$ & $0.48 \pm 1.39$ & $\mathbf{0 . 0 0 2}$ \\
Predicted (\%) & $78.71 \pm 24.41$ & $84.28 \pm 24.20$ & $5.56 \pm 18.88$ & $\mathbf{0 . 0 0 7}$
\end{tabular}

everything under 0.05 are in bold
ATA 100\% oxygen had no significant effect on FEV1, FEV1/FVC and FEF25-75, and a small though statistically significant improvement in both FVC and PEF. Thus, even though current HBOT protocols include a higher number of daily sessions, they did not result in pulmonary toxicity.

The mean increase in FVC of $2.8 \%$ from baseline after 60 hyperbaric oxygen daily sessions, is relatively small and clinically insignificant. One can claim that the increase can be attributed to training or learning to perform the forced expiratory even though 3 months have elapsed between baseline and post HBOT evaluations. However, if it was attributed to maneuver training, it would have also resulted in an increase in FEV1, which was not seen.. The increase in PEF, which is an effort dependent index, even though found in 3-month interval, may theoretically be related to the learning effect of the patient 
Table 3 Units of pulmonary toxicity dose (UPTD) in HBOT studies

\begin{tabular}{llll}
\hline & $\begin{array}{l}\text { UPTD per } \\
\text { session }\end{array}$ & $\begin{array}{l}\text { Number of } \\
\text { sessions }\end{array}$ & $\begin{array}{l}\text { Total } \\
\text { UPTD }\end{array}$ \\
\hline $\begin{array}{l}\text { Hadanny, Efrati } \\
\text { et al. }\end{array}$ & 224 & 60 & 13,489 \\
Pott et al. & 273 & 30 & 8213 \\
Thorsen et al. & 273 & 21 & 5749 \\
\hline
\end{tabular}

repeating the test. Enright et al. showed a change of $5.7 \%$ inf PEF in repeated tests [16].

Two previous studies had conflicting results regarding the pulmonary toxicity effects of repetitive HBOT sessions. In the Pott et al. study [7] in which 18 patients had 30 daily sessions (Table 3) in a monoplace chamber for $90 \mathrm{~min}$ at 2.4 ATA oxygen without air breaks, no significant changes in FVC or diffusing capacity were noticed. However, the Pott et al. study had a considerably small sample, where only 14 patients completed more than 20 sessions. The standard deviations of pulmonary functions were considerably large (over 20\%), thus small changes could have been missed. The second study done by Thorsen et al. [8], included 20 patients treated in 21 HBOT daily sessions for 90 min of 2.4 ATA with 30: 5 air breaks (Table 3), no significant changes in FVC, FEF25\%, PEF or diffusing capacity were noticed. However, there were significant reductions in FEV1 and FEF50-75\%.

Compared to those studies (Table 3), the current study has two main strengths. First, this is the largest prospective sample size $(N=88)$. Post-hoc sample power analysis found the current study has a power of $93.3 \%$. Second, the currently used protocol is significantly longer (60 sessions) and suitable for the updated clinical use of HBOT.

One of the theories regarding the oxygen toxicity mechanism relies on the generation free radicals, which are the byproducts of the respiratory chain for adenosine triphosphate (ATP) production by the mitochondria. Harabin et al. [17] showed that intermittent delivery of HBOT (relatively long periods of hyperoxia interrupted by short periods of low oxygen pressure or normoxia) reduces pulmonary toxicity in animal models. The mechanism of tolerance is mediated by inducing the lung enzyme superoxide dismutase (SOD), which functions as a free radicals scavenger [17]. Therefore, even though HBOT may increase free radical production, intermittent exposure actually induces SOD and other scavengers that reduce the net free radical concentrations and their potential toxic effects.
Arieli et al. [18] calculated a power equation for pulmonary oxygen toxicity, as measured by vital capacity reduction:

$$
\% \Delta \mathrm{VC}=0.0082 \times \mathrm{t}^{2} \times\left(\mathrm{PO}_{2}\right)^{4.57}
$$

Using our HBOT protocol variables of 2 ATA for 90 $\min$ :

$\Delta \mathrm{VC}=0.4382 \%$ after a single exposure.

Next, the recovery from pulmonary oxygen toxicity of the hyperbaric exposure was calculated using [18]:

$$
\begin{aligned}
\Delta \operatorname{VCtr} \%= & \Delta \mathrm{VCe} \% \\
& \times \mathrm{e}^{-\left(-0.42+3.83927 \times\left(\mathrm{PO}_{2}\right)_{\mathrm{ex}}\right) \times \operatorname{tr}}
\end{aligned}
$$

where $\operatorname{tr}$ is the recovery time in hours. $\Delta \mathrm{VCtr}$ is the value after the recovery time, $\triangle \mathrm{VCe}$ is the value following the previous hyperbaric oxygen exposure, and $\mathrm{PO}_{2} \mathrm{ex}$ is the previous exposure to hyperbaric oxygen in ATA. The rate of recovery depends on the $\mathrm{PO}_{2}$ which caused the insult.

Using a recovery time of $22.5 \mathrm{~h}$ after one hyperbaric session, $\Delta \mathrm{VCtr}$ is zeroed.

Thus, considering our protocol of 60 daily sessions, separated by $\sim 24 \mathrm{~h}, \Delta \mathrm{VC}$ results in a complete recovery between sessions. Moreover, using daily sessions at $90 \mathrm{~min}$ of 2 ATA, no pulmonary toxicity is expected regardless of the number of sessions. In addition to vital capacity recover, as discussed previously, the levels of scavengers enzymes might still be higher than normal.

The current study has several limitations, First, no control group was used. However, a previous study on 46 healthy males that evaluated the test re-test variability over a period of 3 months, showed that there were no significant changes in FEV1, FVC, FEV1/FVC, PEF and FEF25-75 [19]. Second, lung capacity and diffusion tests were not performed in the study. In the previous studies mentioned above $[7,8]$, there were no changes in these functions. Third, patients suffering from significant chronic lung diseases were not included in the study. Fourth, spirometry is not sensitive enough to detect small (alveolar) changes. Fifth, the baseline weight and height of the patients were used for all measurements.

\section{Conclusions}

With regards to pulmonary functions, the currently used HBOT protocol that includes 60 daily sessions of $90 \mathrm{~min}$ exposure to $100 \%$ oxygen at 2 ATA, with 5 min air breaks every $20 \mathrm{~min}$, has no negative effects on pulmonary functions. Surprisingly, there was a modest non clinically significant thought statistically significant improvement in PEF and FVC in the current cohort of patients without chronic lung disease. Further studies are needed for patients with lung diseases. 


\section{Abbreviations}

ATA: Absolute atmospheres; FEF25\%: Forced expiratory flow at 25\% of pulmonary volume; FEF25-75\%: Forced mid-expiratory flow rate; FEV1: Forced expiratory volume in one second; FVC: Forced vital capacity: HBOT: Hyperbaric oxygen therapy; PEF: Peak expiratory flow rate

\section{Acknowledgements}

Not Applicable.

\section{Authors' contributions}

Authorship contributions: $\mathrm{AH}=$ designed, interpreted, analyzed the data and drafted the manuscript. SE = designed, interpreted, analyzed the data and drafted the manuscript. $\mathrm{ZT}=$ acquired, interpreted and analyzed the data, approved the manuscript TAL = acquired interpreted, analyzed the data and approved the manuscript. BY = acquired the data and approved the manuscript, $\mathrm{FG}=$ acquired the data and approved the manuscript, $\mathrm{LE}=$ acquired the data and approved the manuscript, $\mathrm{PN}=$ acquired the data and approve the manuscript, $\mathrm{BJ}=$ acquired the data and approved the manuscript, FM = acquired the data and approved the manuscript. All authors have agreed both to be personally accountable for the author's own contributions and to ensure that questions related to the accuracy or integrity of any part of the work.

\section{Funding}

No funding was received for this study.

\section{Availability of data and materials}

The datasets used and/or analysed during the current study are available from the corresponding author on reasonable request.

\section{Ethics approval and consent to participate}

The protocol was approved by Assaf Harofeh Medical Center review board (Helsinki committee) (IRB) (0024-16-ASF). All participants signed written informed consent prior to their inclusion.

\section{Consent for publication}

Not Applicable.

\section{Competing interests}

The authors declare that they have no competing interests.

\section{Author details}

${ }^{1} T$ The Sagol Center for Hyperbaric Medicine and Research, Shamir Medical Center, Zerifin, Israel. ${ }^{2}$ Sackler School of Medicine, Tel-Aviv University, Tel-Aviv, Israel. ${ }^{3}$ Bar Ilan University, Ramat-Gan, Israel. ${ }^{4}$ Faculty of Biomedical Engineering, Technion, Haifa, Israel. ${ }^{5}$ Research and Development Unit, Shamir Medical Center, Zerifin, Israel. ${ }^{6}$ Sagol School of Neuroscience, Tel-Aviv University, Tel-Aviv, Israel.

Received: 29 January 2019 Accepted: 10 July 2019

Published online: 13 August 2019

\section{References}

1. Thomson L, Paton J. Oxygen Toxicity; 2014.

2. Clark JM, Lambertsen CJ. Pulmonary oxygen toxicity: a review. Pharmacol Rev. 1971;23(2):37-133.

3. van Ooij PJ, Hollmann MW, van Hulst RA, Sterk PJ. Assessment of pulmonary oxygen toxicity: relevance to professional diving; a review. Respir Physiol Neurobiol. 2013;189(1):117-28.

4. Clark JM. Pulmonary limits of oxygen tolerance in man. Exp Lung Res. 1988; 14 Suppl:897-910.

5. Clark JM, Lambertsen CJ. Rate of development of pulmonary $\mathrm{O} 2$ toxicity in man during O2 breathing at 2.0 Ata. J Appl Physiol. 1971;30(5):739-52

6. Clark JM, Lambertsen CJ, Gelfand R, Flores ND, Pisarello JB, Rossman MD, et al. Effects of prolonged oxygen exposure at 1.5, 2.0, or 2.5 ATA on pulmonary function in men (predictive studies V). J Appl Physiol. 1999;86(1): 243-59.

7. Pott F, Westergaard P, Mortensen J, Jansen EC. Hyperbaric oxygen treatment and pulmonary function. Undersea Hyperb Med. 1999;26(4):225-8.

8. Thorsen E, Aanderud L, Aasen TB. Effects of a standard hyperbaric oxygen treatment protocol on pulmonary function. Eur Respir J. 1998;12(6):1442-5.
9. LE W. Hyperbaric oxygen therapy indications. 13th ed. UHMS; 2008. p. 139-152, 283-287. https://www.uhms.org/images/indications/UHMS_HBO2_ Indications_13th_Ed._Front_Matter_References.pdf.

10. Boussi-Gross R, Golan H, Fishlev G, Bechor Y, Volkov O, Bergan J, et al. Hyperbaric oxygen therapy can improve post concussion syndrome years after mild traumatic brain injury - randomized prospective trial. PLoS One. 2013;8(11):e79995.

11. Efrati S, Fishlev G, Bechor Y, Volkov O, Bergan J, Kliakhandler K, et al. Hyperbaric oxygen induces late neuroplasticity in post stroke patients-randomized, prospective trial. PLoS One. 2013;8(1):e53716.

12. Mukherjee A, Raison M, Sahni T, Arya A, Lambert J, Marois P, et al. Intensive rehabilitation combined with $\mathrm{HBO} 2$ therapy in children with cerebral palsy: a controlled longitudinal study. Undersea Hyperb Med. 2014;41(2):77-85.

13. Efrati S, Golan H, Bechor Y, Faran Y, Daphna-Tekoah S, Sekler G, et al. Hyperbaric oxygen therapy can diminish fibromyalgia syndrome--prospective clinical trial. PLoS One. 2015;10(5):e0127012.

14. Miller MR, Hankinson J, Brusasco V, Burgos F, Casaburi R, Coates A, et al. Standardisation of spirometry. Eur Respir J. 2005;26(2):319-38.

15. Knudson RJ, Lebowitz MD, Holberg CJ, Burrows B. Changes in the normal maximal expiratory flow-volume curve with growth and aging. Am Rev Respir Dis. 1983;127(6):725-34.

16. Enright PL, Beck KC, Sherrill DL. Repeatability of spirometry in 18,000 adult patients. Am J Respir Crit Care Med. 2004;169(2):235-8.

17. Harabin AL, Survanshi SS, Weathersby PK, Hays JR, Homer LD. The modulation of oxygen toxicity by intermittent exposure. Toxicol Appl Pharmacol. 1988:93(2):298-311.

18. Arieli R, Yalov A, Goldenshluger A. Modeling pulmonary and CNS O (2) toxicity and estimation of parameters for humans. J Appl Physiol. 2002;92(1): $248-56$.

19. Singha Roy A, Bandyopadhyay A. Pulmonary function of young Muslim males during the month of Ramadan. Am J Mens Health. 2018;12(4):828-36.

\section{Publisher's Note}

Springer Nature remains neutral with regard to jurisdictional claims in published maps and institutional affiliations.
Ready to submit your research? Choose BMC and benefit from:

- fast, convenient online submission

- thorough peer review by experienced researchers in your field

- rapid publication on acceptance

- support for research data, including large and complex data types

- gold Open Access which fosters wider collaboration and increased citations

- maximum visibility for your research: over $100 \mathrm{M}$ website views per year

At $\mathrm{BMC}$, research is always in progress.

Learn more biomedcentral.com/submissions 